\title{
Impact of the COVID-19 Pandemic on Cancer Clinical Trials
}

\author{
Judy C. Boughey, MD, FACS ${ }^{1}$, Rebecca A. Snyder, MD, MPH, FACS ${ }^{2}$, Olga Kantor, MD, MS ${ }^{3}$, \\ Linda Zheng, BS, BA ${ }^{4}$, Akhil Chawla, MD ${ }^{5}$, Toan T. Nguyen, MD, FACS ${ }^{6}$, Shauna L. Hillman, MS ${ }^{7}$, \\ Olwen M. Hahn, $\mathrm{MD}^{8}$, Sumithra J. Mandrekar, $\mathrm{PhD}^{7}$, and Christina L. Roland, MD, MS, FACS ${ }^{9}$ \\ ${ }^{1}$ Department of Surgery, Mayo Clinic, Rochester, MN; ${ }^{2}$ Division of Surgical Oncology, East Carolina University Brody \\ School of Medicine, Greenville, NC; ${ }^{3}$ Division of Breast Surgery, Department of Surgery, Brigham and Women's \\ Hospital, Boston, MA; ${ }^{4}$ American College of Surgeons Cancer Research Program, Chicago, IL; ${ }^{5}$ Division of Surgical \\ Oncology, Department of Surgery, Northwestern Medicine Regional Medical Group, Northwestern University Feinberg \\ School of Medicine, Chicago, IL; ${ }^{6}$ Lakeland Regional Health, Lakeland, FL; ${ }^{7}$ Department of Health Sciences Research, \\ Alliance Statistics and Data Management Center, Mayo Clinic, Rochester, MN; ${ }^{8}$ Alliance Protocol Operations Office, \\ University of Chicago, Chicago, IL; ${ }^{9}$ Department of Surgical Oncology, University of Texas MD Anderson Cancer Center, \\ Houston, TX
}

\begin{abstract}
The COVID-19 pandemic has had widespread impact on healthcare, resulting in modifications to how we perform cancer research, including clinical trials for cancer. The impact of some healthcare workers and study coordinators working remotely and patients minimizing visits to medical facilities impacted clinical trial participation. Clinical trial accrual dropped at the onset of the pandemic, with improvement over time. Adjustments were made to some trial protocols, allowing telephone or video-enabled consent. Certain study activities were permitted to be performed by local healthcare providers or at local laboratories to maximize patients' ability to continue on study during these challenging times. We discuss the impact of COVID-19 on cancer clinical trials and changes at the local, cooperative group, and national level.
\end{abstract}

The onset of the COVID-19 pandemic in the USA in early 2020 triggered a drastic reprioritization of healthcare resources across the nation. Hospitals and healthcare personnel prepared for one of the worst pandemics in US history, while research efforts shifted focus toward testing

(C) Society of Surgical Oncology 2021

First Received: 7 April 2021

Accepted: 24 June 2021;

Published Online: 8 July 2021

J. C. Boughey, MD, FACS

e-mail: Boughey.judy@mayo.edu experimental therapies to treat critically ill patients and developing a vaccine against the novel coronavirus. Patients with cancer, especially those undergoing active treatment, are at increased risk of severe illness from COVID-19. Therefore, it was clear from the start of the pandemic that modifications to cancer research, particularly clinical trials, would be necessary.

\section{CLINICAL TRIAL ACCRUAL AND CONDUCT}

The COVID-19 outbreak caused tremendous disruption to cancer clinical trial accrual and conduct. A survey at the end of March 2020 suggested that at least $60 \%$ of investigators had halted or delayed screening and enrollment of patients to clinical trials, and $50 \%$ had reprioritized enrollment to certain higher-priority trials.' A small global survey suggested that $20 \%$ of US respondents had completely stopped new patient enrollment and 60\% were enrolling at a lower than usual rate. ${ }^{2}$ The SWOG Cancer Research Network from January through April 2020 showed a direct correlation between the uptick of COVID19 cases across the USA and subsequent decrease of clinical trial enrollment by approximately $50 \%{ }^{3}$

The decline in accrual to cancer clinical trials during the COVID-19 pandemic was multifactorial. Many institutions modified their clinical trial programs early in the pandemic with the suspension of elective diagnostic and surgical procedures. Thus, it is not surprising that a significant reduction in the number of patients diagnosed with cancer was evident, with a $46.4 \%$ decrease in cancer diagnoses 
when combining the six most common malignancies in the $\mathrm{USA}^{4}$, ranging from a $24.7 \%$ drop in patients diagnosed with new pancreatic cancer to as much as a $51.8 \%$ drop in new breast cancer diagnoses. This decline was likely a direct consequence of the reduction in screening and diagnostic imaging procedures and biopsies, such as the $94 \%$ drop in mammographic screening.

Disruptions directly impacted ability to obtain and review research biopsies, which also directly affected clinical trial enrollment. In most places, research biopsies were stopped when elective procedures stopped, and since many research laboratories were closed, the ability to process research tissue and blood was severely limited.

During the early months of the pandemic, there was substantial repurposing of medical support staff. Those deemed "nonessential personnel" were redeployed, transitioned to working remotely, or furloughed, creating immediate gaps in clinical trial operations. The rapidity with which these changes occurred gave institutions, providers, and patients limited time and resources to adapt to significant structural changes and impact on research protocols. This led to a lack of on-site research coordinator support, resulting in a decrease in clinical trial enrollment across the nation. Even during the initial COVID-19 recovery process, many institutions have continued to limit on-site research staff and restrict use of clinic staff for research due to social distancing requirements.

Together, these constraints have translated to an increased workload for physicians and clinicians, who in many cases have taken on the burden of screening and consenting patients for clinical trials during patient care activities. Additionally, analytical support for ongoing clinical trial activities was also affected by the redeployment and repurposing of statistics and data center personnel, including trial data managers, statisticians, and registration and information technology (IT) support.

On top of these modifications made at the institution level, patients and their caregivers had to consider exposure risk when traveling to and from medical centers for trial participation. Some patients who required long-distance travel to tertiary referral centers could even face state mandates for self-quarantine when crossing state lines. Many patients opted for treatment options that required fewer visits to medical centers and/or less travel, which often precluded clinical trial consideration. For patients in trials necessitating travel to receive investigational agents or for patients with high-risk comorbidities, clinical trialists were encouraged to consider and document dose delays or omissions, postpone or cancel research biopsies, and utilize local laboratory testing for within-cycle monitoring.

\section{MODIFICATIONS TO THE INFORMED CONSENT PROCESS}

The informed consent process has always been the central element in maintaining ethical standards in clinical trials by protecting the autonomy of human subjects. However, the COVID-19 pandemic presented unique challenges to this critical step because it exposed the research team and potentially vulnerable patients to additional opportunities for COVID-19 transmission. Many trial protocols that required face-to-face visits to discuss risks and benefits and ultimately obtain consent were suspended.

Alternative pathways to the face-to-face consent process, such as telephone or video-enabled consent, have been explored previously. ${ }^{6}$ Employing telemedicine capability for the informed consent process can be effective and may enable a rebound in clinical trial participation. The US Food and Drug Administration (FDA) has published guidance on the use of electronic informed consents in lieu of standard written consents, which can be utilized inperson or in remote circumstances. ${ }^{7}$ Digital consents have been previously shown to increase participation among rural and minority populations, both patient populations traditionally underrepresented in clinical trials. It should also be noted that the National Cancer Institute (NCI) Central Institutional Review Board (CIRB) supports remote informed consent and does not consider this to be a protocol deviation. The remote informed consent process allows for phone or video consent discussion with the patient, followed by completion of a written consent form that is mailed or electronically sent to the patient for signature and return.

\section{INSTITUTIONAL REVIEW BOARD (IRB)}

Institutional review boards (IRBs) have the primary duty to protect the welfare, rights, and privacy of human subjects undergoing any clinical trial. Appropriately, studies related to COVID-19 have been prioritized. IRBs, clinical trial development and research support, and other institutional resources were devoted first to COVID-19 studies, temporarily sidelining routine oncologic trials. Unfortunately, this has resulted in a tremendous backlog in study startup at many sites, ultimately leading to significant delays in opening new cancer-related clinical trials. Other research infrastructural support functions not viewed as critical to patient care include processes vital to clinical trial success, such as regulatory affairs, budget negotiation, and legal contracting, which have all seen significant delays secondary to the pandemic. 
The FDA also issued specific guidance to investigators and IRBs regarding the conduct of clinical trials during the COVID-19 pandemic, addressing situations in which patients' interests are best served by continuing as trial participants per protocol as well as situations in which withdrawal is more appropriate, such as the need for ongoing safety monitoring, the impact of a specific investigational product, or the nature of the disease. Risk mitigation during the COVID-19 pandemic is a dynamic process and is dependent on the geographical intensity of the pandemic, institutional safeguards and resources, and the risk-benefit ratio of participation in trials.

\section{DISPARITIES IN CLINICAL TRIAL ACCRUAL}

It is well established that fewer Black patients or patients of minority race are accrued to cancer clinical trials compared with patients of white race. According to one recent study of clinical trial enrollment by race, Black patients accounted for only $3 \%$ of industry-sponsored trials and $9 \%$ of SWOG trials, while accounting for $12 \%$ of the US population with cancer. ${ }^{10}$ It has been clearly demonstrated that Black and minority populations have higher incidence of hospitalization and death from COVID-19 infection. 'Given the already-existing disparity in minority clinical trial enrollment, combined with higher rates of COVID-19 infection among minority populations, an exacerbation in racial disparities in cancer clinical trial enrollment and health outcomes is expected.

Although not yet well understood, it is likely that COVID-19 has affected availability and accrual to clinical trials in urban and rural centers differently. Many rural oncology practices lack adequate research support and clinical trial resources when compared with larger healthcare systems and comprehensive cancer centers. As a result, these sites may be disproportionately affected by both rising rates of COVID-19 infections and lack of support to keep clinical trials open, further intensifying rural cancer disparities.

While many large academic institutions may have been able to withstand the increased regulatory and financial burden imposed by COVID-19, smaller community centers-many of which have already faced challenges in clinical trials enrollment and conduct-are likely to be disproportionately impacted.

\section{NATIONAL CANCER INSTITUTE GUIDANCE}

The National Cancer Institute (NCI) provided interim guidance for clinical trials supported through the NCI Cancer Therapy Evaluation Program (CTEP) and the NCI Community Oncology Research Program (NCORP) on 13
March 2020, early in the COVID-19 pandemic. ${ }^{13}$ The goal was to enable sites to continue to provide care to patients enrolled on NCI clinical trials and to mitigate potential immediate hazard for patients. These changes included allowances for transfer of a patient's care to a different participating site and continuity of care to be provided by nonresearch staff. The latter provision allowed a local healthcare provider to conduct certain study activities when the patient could not travel to the enrolling study site. This has been implemented on a short-term basis and is focused on situations in which it is in the patient's best interest to remain on a study, and includes laboratory testing, imaging, drug therapy with noninvestigational agents, standard radiation, surgery, and other interventions that do not require protocol-specified credentialing. For studies including oral investigational agents, shipping drug directly to the patient was also allowed.

The NCI released additional guidance on 23 March 2020 to address alternative procedures that were either minor or major protocol deviations. ${ }^{14}$ Minor protocol deviations included study visits conducted by phone or videoconference, including adverse event assessment; delayed study visits, laboratory testing, or imaging; and missed study visits if benefit of delaying the visit outweighed risk of exposure. Treatment delays that did not impact patient safety or compromise study integrity and certain alterations to biospecimen collection were considered minor deviations. Major protocol deviations were defined as alternative procedures that impacted patient safety, substantially altered risks to the patient, compromised the overall integrity of the study data (ability to draw conclusions from the study data), or affected the willingness of the patient to participate in the trial. In addition, on-site auditing and monitoring of trials were rescheduled, and many transitioned to remote audits.

NCI required the National Clinical Trial Network (NCTN) groups to collect all minor protocol deviations due to COVID-19 to facilitate central IRB reporting. The Alliance for Clinical Trials in Oncology elected to implement this by creating a single Rave database, ALLIANCE_COVID-19-Reporting, for the centralized collection of all COVID-19 pandemic-related events, including COVID-19 testing and outcome, discontinuation of study treatment, and termination of study follow-up due to COVID-19.

\section{CORRELATION WITH SURGICAL ACTIVITY}

Although precise data regarding delays or disruptions in surgical oncology care during the COVID-19 pandemic across institutions participating in NCTN clinical trials are unknown, a systematic review of international and US 
FIG. 1. Weekly enrollment to Alliance trials from 1 February 2020 to 28 February 2021

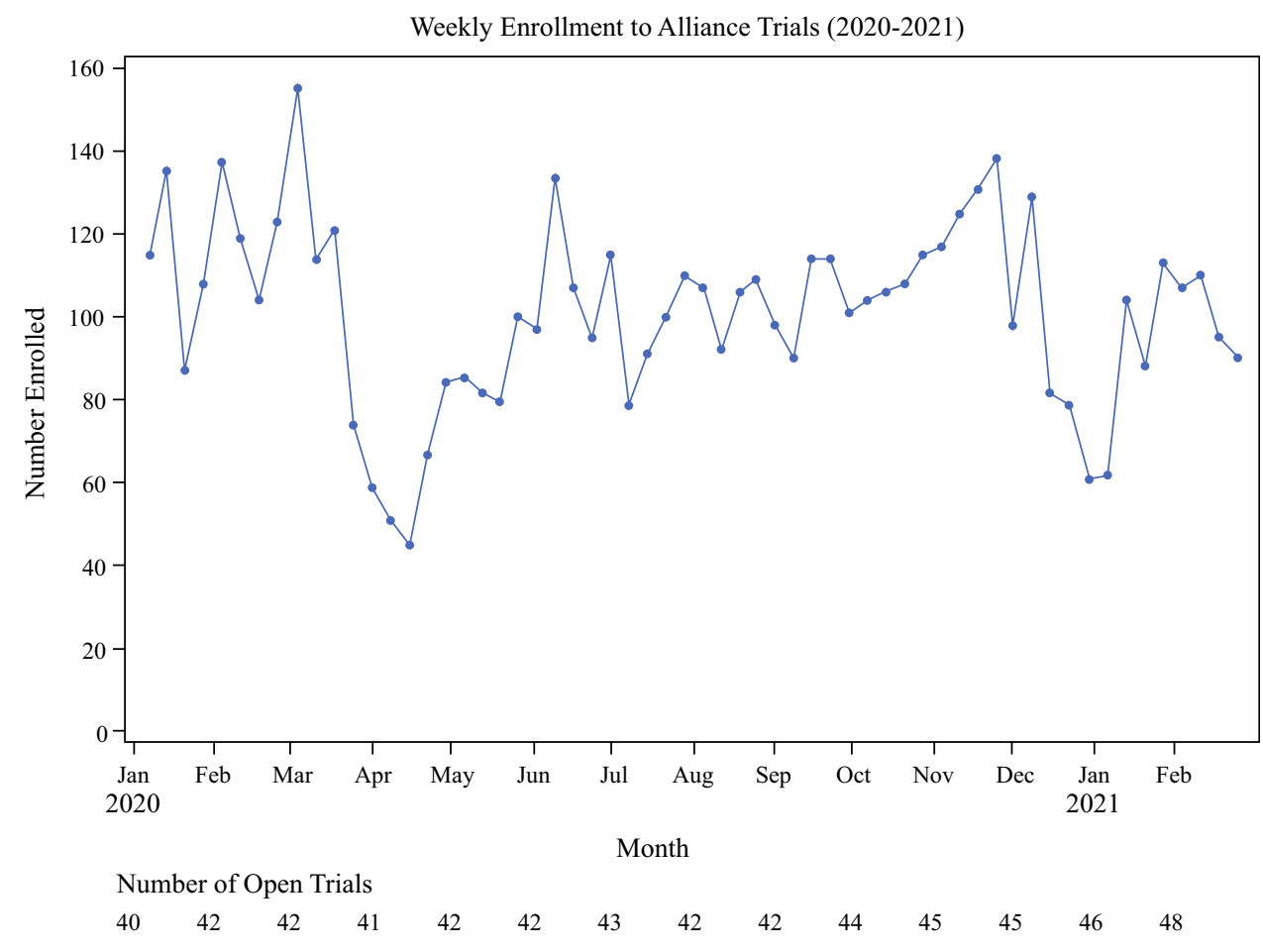

studies demonstrated a broad range in the extent of delays and disruptions in surgery (reported by $3-76 \%$ of survey respondents) and reduction in the number of operations (14-86\%) during the pandemic. ${ }^{15}$ Although it is difficult to assess directly the relationship of disruptions in surgical care and clinical trial activity, it is likely that these are interconnected.

\section{RESTARTING CLINICAL TRIALS}

Declining enrollment in cooperative group trials illustrates the impact of COVID-19 on cancer clinical trials across the nation. Between March and May 2020, there was a significant decrease in accrual to clinical trials in the Alliance for Clinical Trials in Oncology to less than half the usual weekly enrollment figures (Fig. 1). Recovery in accrual was gradual, with return to baseline rates in the fall. There was another dip in accrual rates over the weeks of the holiday season, which is commonly seen.

Although the initial shutdown for many clinical trials was sudden, restarting required a phased approach to protect the safety of the patients as well as the healthcare providers, while allowing patients the opportunities afforded by the clinical trials to continue. As the country recovered from the initial impact of the pandemic, trials began to reopen in a stepwise fashion, beginning with studies designed for patients lacking further treatment options outside of a clinical trial (i.e., phase I trials). During the next phase, trials that provided access to potentially life-prolonging or life-saving interventions were reopened. Over time, many institutions also began to allow enrollment in trials as long as additional in-person visits, research biopsies, interventions, or personal protective equipment were not required. Subsequently, trials with a potentially therapeutic impact that required research biopsies, blood draws, and in-person visits reopened. Observational cohort studies, tissue collection studies, and quality-of-life studies were the last trials to resume.

While the COVID-19 pandemic has not yet ended, restrictions to clinical trial conduct have been lifted. Moving forward, it is anticipated that there will be the continued need for a hybrid system of virtual visits along with in-person aspects of clinical trials, which may ultimately result in unintended but meaningful improvements in clinical trial conduct and accessibility.

In the setting of COVID-related economic unrest and decreased federal and philanthropic funding for cancer programs, cancer research funding and philanthropy have decreased considerably. Large organizations that currently contribute to cancer clinical trial funding, such as the American Cancer Society and Susan G. Komen, have seen significant decreases in donations, which has led to the deferment of grant cycles during the pandemic. ${ }^{16,17}$

Clinical trialists must adapt to a smaller resource pool by designing trials that lessen financial burden on clinical trial offices and institutions. Designing new ways by which data are obtained in a real-world setting may lessen the financial burden of running a large-scale trial. An example 
is the novel concept of the ICAREdata ${ }^{\circledR}$ project, which enables easier prospective data gathering and has teamed with Alliance for Clinical Trials in Oncology to capture data within the medical record that is already being captured for clinical purposes. ${ }^{18}$ In addition, identifying methods to enable industry contribution to trial processes while limiting influence on what is being studied will be important. Finally, clinicians and cancer researchers alike must engage industry and community partners and organizations to change the landscape in foundation and philanthropic funding for clinical trials. Diligent reassessment of priorities and advocacy for cancer programs will be essential as we eventually move beyond COVID-19 recovery and into a new normal for cancer programs.

The COVID-19 pandemic led to an abrupt change in the use of telehealth in clinical practice and for clinical trials. While the rapid implementation of telehealth was accompanied by a number of challenges, a digital care delivery model of some type will likely be integrated in the future of cancer care, including the conduct of clinical trials. This may yield potential improvements, as the classic clinical trial process is frequently cumbersome and limits clinical trial access to select subgroups of patients, often those with more socioeconomic advantage. Telehealth may decrease the need for patients to travel to a clinical trial site for consent, testing, and appointments and lead to improved accrual and data regarding adverse effects and follow-up information. Overall, this may ultimately increase availability of clinical trials to more diverse patient populations, especially those limited by travel distance and transportation barriers.

FUNDING Research reported in this publication was supported by the National Cancer Institute of the National Institutes of Health under award number U10CA180882 (to the Alliance for Clinical Trials in Oncology Statistics and Data Management Center Health). $\mathrm{h}$ ttps://acknowledgments.alliancefound.org.

\section{DECLARATIONS}

DISCLOSURE C.R.: Bristol Myers Squibb-Research funding

\section{REFERENCES}

1. Waterhouse DM, Harvey RD, Hurley P, et al. Early impact of COVID-19 on the conduct of oncology clinical trials and longterm opportunities for transformation: findings from an American Society of Clinical Oncology survey. JCO Oncol Pract. 2020;16(7):417-21.

2. Upadhaya S, Yu JX, Oliva C, Hooton M, Hodge J, HubbardLucey VM. Impact of COVID-19 on oncology clinical trials. Nat Rev Drug Discov. 2020;19(6):376-7.

3. Unger JM, Blanke CD, LeBlanc M, Hershman DL. Association of the coronavirus disease 2019 (COVID-19) outbreak with enrollment in cancer clinical trials. JAMA Netw Open. 2020;3(6):e2010651.
4. Kaufman HW, Chen Z, Niles J, Fesko Y. Changes in the number of US patients with newly identified cancer before and during the coronavirus disease 2019 (COVID-19) pandemic. JAMA Netw Open. 2020;3(8):e2017267.

5. Naidich JJ, Boltyenkov A, Wang JJ, Chusid J, Hughes D, Sanelli PC. Impact of the coronavirus disease 2019 (COVID-19) pandemic on imaging case volumes. $J$ Am Coll Radiol. 2020;17(7):865-72.

6. Welch BM, Marshall E, Qanungo S, et al. Teleconsent: a novel approach to obtain informed consent for research. Contemp Clin Trials Commun. 2016;3:74-9.

7. US Food and Drug Administration. Use of Electronic Informed Consent in Clinical Investigations - Questions and Answers: Guidance for Institutional Review Boards, Investigators, and Sponsors. Published December 15, 2016. Accessed September 13, 2020. https://www.fda.gov/media/116850/download

8. Clark D, Woods J, Patki D, et al. Digital informed consent in a rural and low-income population. JAMA Cardiol. 2020;5(7):845-7.

9. US Food and Drug Administration. FDA Guidance on Conduct of Clinical Trials of Medical Products during COVID-19 Public Health Emergency: Guidance for Industry, Investigators, and Institutional Review Boards. Published March 2020 (Updated on April 16, 2020). Accessed September 13, 2020. https://www.fda.g ov/regulatory-information/search-fda-guidance-documents/fda-g uidance-conduct-clinical-trials-medical-products-during-covid-1 9-public-health-emergency

10. Unger JM, Hershman DL, Osarogiagbon RU, et al. Representativeness of Black patients in cancer clinical trials sponsored by the National Cancer Institute compared with pharmaceutical companies. JNCI Cancer Spectr. 2020;4(4):pkaa034.

11. Centers for Disease Control and Prevention. COVID-19 Hospitalization and Death by Race/Ethnicity. Updated August 18, 2020. Accessed September 1, 2020. https://www.cdc.gov/corona virus/2019-ncov/covid-data/investigations-discovery/hospitalizati on-death-by-race-ethnicity.html

12. Paul R, Arif AA, Adeyemi O, Ghosh S, Han D. Progression of COVID-19 from urban to rural areas in the united states: a spatiotemporal analysis of prevalence rates. $J$ Rural Health. 2020;36(4):591-601.

13. National Cancer Institute. Interim Guidance for Patients on Clinical Trials Supported by the NCI Cancer Therapy Evaluation Program and the NCI Community Oncology Research Program (NCORP). Published March 13, 2020. Accessed September 6, 2020. https://ctep.cancer.gov/content/docs/Memorandum_on_Int erim_Guidance_for_Clinical_Trial_Activities_Affected_by_the_ Novel_Coronavirus-3-13-2020.pdf

14. National Cancer Institute. Additional Guidance Regarding Alternative Procedures for Clinical Trials Supported by the NCI Cancer Therapy Evaluation Program (CTEP) and NCI Community Oncology Research Program (NCORP) Affected by the Spread of the Novel Coronavirus. Published March 23, 2020. Accessed September 6, 2020. https://ctep.cancer.gov/investigato rResources/docs/Memorandum_on_Additional_Guidance_for_Cl inical_Trial_Activities_Affected_by_the_Novel_Coronavirus_323-2020.pdf

15. Riera R, Bagattini AM, Leite Pacheco R, Vianna Pachito D, Roitberg F, Ilbawi A. Delays and disruptions in cancer health care due to COVID-19 pandemic: systematic review. JCO Glob Oncol. 2021;7:311-23.

16. American Cancer Society. COVID-19 and 2020 ACS Grants. Accessed August 31, 2020. https://www.cancer.org/research/we-f und-cancer-research/apply-research-grant/grant-types/covid-19-a nd-2020-acs-grants.html

17. Cahan, E. COVID-19 cancels charity galas and walks. Science is paying the price. Published June 24, 2020. Accessed August 31, 
2020. https://www.sciencemag.org/news/2020/06/covid-19-cance 1s-charity-galas-and-walks-science-paying-price

18. Bertagnolli MM, Anderson B, Quina A, Piantadosi S. The electronic health record as a clinical trials tool: opportunities and challenges. Clin Trials. 2020;17(3):237-42.
Publisher's Note Springer Nature remains neutral with regard to jurisdictional claims in published maps and institutional affiliations. 